\title{
The AIBE and the new phase of the RBHH
}

Ângelo Maiolino

Departamento de Clínica Médica, Universidade Federal do Rio de Janeiro - UFRJ, Rio de Janeiro (RJ) Brazil

President, Associazione Italo-Brasiliana Di Ematologia

Submitted: 2/14/2011

Accepted: 2/14/2011

Correspondence: Ângelo Maiolino Hospital Universitário Clementino Fraga Filho

Rua Professor Rodolpho Paulo Rocco, 255 Serviço de Hematologia, $4^{\circ}$ andar, sala 4A12

Cidade Universitária, Ilha do Fundão

21941-913 - Rio de Janeiro (RJ), Brazil

Phone: 5521 - 25622462

Mobile: 55 21-81116663

maiolino@hucff.ufrj.br

www.rbhh.org or www.scielo.br/rbhh

DOI: 10.5581/1516-8484.20110003
The Associação Ítalo-Brasileira de Hematologia (Italian-Brazilian Association of Hematology - AIBE) was founded on 04/02/2004 in the city of Genoa, Italy. The primary objectives of the AIBE are to promote the integration of the hematology service of both countries through an exchange of healthcare professionals and the development of research of common interest.

Since its inception, the AIBE chose the Revista Brasileira de Hematologia e Hemoterapia (RBHH) as its official vehicle for the publication of scientific articles. For us, it is a great pleasure to participate in this new phase of the journal, its internationalization.

The AIBE can be a powerful tool in this process, as publishing the journal in English and its future indexing by Pubmed will increase, without any doubt whatsoever, the interest of colleagues and Italian researchers in publishing their articles in the journal.

It is important to note that at present Italy is ranked second in the world in the number of scientific publications in the field of hematology. The Italian members, who helped found the AIBE are also in the leadership and participate in several cooperative groups that develop highly important research protocols. Today, cooperation and multicenter studies are of fundamental importance in the development of medical science and Italy has a long tradition and has advanced greatly in recent years in the development of these studies.

The AIBE and the RBHH are partners in this process and we believe that there will be a great synergy in actions that undoubtedly will lead to great steps forward for both. 